\title{
O EFEITO CASIMIR
}

\author{
Yuri Alves De Oliveira ${ }^{1}$, Mauro Antonio Andreata 2 . \\ Departamento de Física, Universidade Federal de Goiás - Campus Catalão \\ Caixa Postal 536, 75704-020 Catalão, GO \\ yurialves.y@gmail.com ${ }^{1}$, mauroandreata@yahoo.com.br ${ }^{2}$
}

\begin{abstract}
In this work we present the Casimir effect proposed by the Dutch physicist Hendrik Casimir in 1948, where two parallel plates and electrically neutral, in a region where there is a vacuum, separated by a distance of microns suffer the action of an attractive force. Afterwards we present how the evolution of the concepts related to the vacuum occurred, ranging from classical to quantum, showing the contribution of many scientists and researchers throughout history. Finally, we explain how the quantum vacuum, associated with zero-point energy, can be used to understand the Casimir effect and how was the trajectory of experimental verification of this phenomenon.
\end{abstract}

Keywords—Effect Casimir, Vacuum, Zero-point energy;

Resumo- Neste trabalho apresentamos o Efeito Casimir, proposto pelo físico holandês Hendrik Casimir, em 1948, onde duas placas paralelas e eletricamente neutras, em uma região onde há somente o vácuo, separadas por uma distância de micrômetros, sofrem a ação de uma força de caráter atrativo. Discutimos como ocorreu a evolução dos conceitos relacionados ao vácuo, desde o clássico ao quântico, mostrando a contribuição de vários cientistas e pesquisadores ao longo da história. Por fim, expomos como o vácuo quântico, associado a energia de pontozero, pode ser utilizado para compreender o Efeito Casimir e como foi a trajetória de verificação experimental deste fenômeno.

Palavras-chave—Efeito Casimir, Vácuo, Energia de ponto zero;

\section{Introdução}

Suponha o seguinte sistema (Figura1): duas placas metálicas paralelas separadas por uma distância de micrômetros e colocadas no interior de uma câmara, com um dispositivo sensível o suficiente para medir qualquer tipo de força, onde o vácuo se faz presente. Suponha ainda que estas placas sejam eletricamente neutras, de forma que não há forças elétricas entre elas, e que a força de gravidade é desprezível e suponha ainda que tal sistema seja construído de tal maneira que quaisquer forças externas possam ser precisamente neutralizadas. Espera-se, diante destas circunstâncias, que o dispositivo não acuse a existência de qualquer força no interior da câmara; entretanto, contrariando tal expectativa não é que o que ocorre! Surpreendentemente, o dispositivo indica a presença de uma força de atração entre as placas.

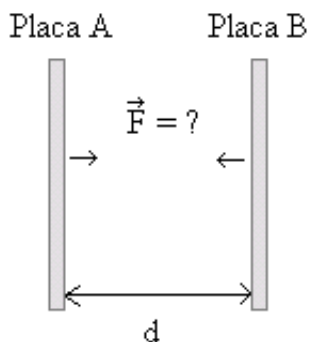

Figura 1. O Efeito Casimir.
No ano de 1948, o físico Hendrik Brugt Gerhard Casimir (1909 - 2000) previu este fenômeno teoricamente ${ }^{[1]}$, o que foi posteriormente denominado Efeito Casimir justamente em sua homenagem. Para duas placas separadas por uma distância de um micrômetro e com áreas transversais de um centímetro quadrado, segundo a previsão de Casimir, a força deve ser de 0,013 dinas, ou o equivalente $1,3 \times 10^{-7}$ Newtons.

Como veremos, o Efeito Casimir está intimamente ligado ao vácuo quântico e às flutuações do campo eletromagnético devido à energia de ponto zero, mas antes disso apresentaremos como o conceito de vácuo evoluiu no decorrer da história, desde o clássico ao quântico.

\section{Do Vácuo Clássico ao Quântico}

O conceito clássico de vácuo vem do latim $v a$ cuит que significa literalmente vazio. Na Física clássica o vácuo é tido como o espaço na ausência de matéria e de energia e seu conceito ou mesmo sua "existência" foi alvo de um longo processo de discussão entre diversos cientistas e entusiastas. Desde a antiguidade há dificuldades consideráveis em entender o conceito do vazio absoluto, do vácuo, e as ideias desenvolvidas até o presente momento sofreram diversas reviravoltas.

$\mathrm{Na}$ antiguidade clássica, na Grécia por volta do século V a. C, Parmênides (ca. 530 - 460 a. C) defendia a ideia que todo o Universo era preenchido 
por uma espécie de substância, o "ser" [2], e segundo ele era impossível existir o "não ser", isto é, a ausência de todas as coisas. Para este filósofo toda a realidade é imutável e o nada, o "não ser" absoluto, seria algo impossível uma vez que tudo "é", de forma que o nada seria uma contradição lógica. Um discípulo de Parmênides, Zenão de Eleia (ca. 490 - 430 a. C), conhecido por seus paradoxos e a crença de que o movimento era uma ilusão provocada pelos nossos sentidos, defende a ideia da impossibilidade do vácuo proposta por seu mestre que acreditava ser o movimento um deslocamento para o vácuo e como este não existia então o movimento seria impossível [2].

Para os atomistas, por volta de 430 a. C, como Leucipo (ca. 480 - 420 a. C) e Demócrito (ca. 460 370 a. C) tudo era composto de átomos, do grego "indivisível". Para estes filósofos, o átomo era a menor parte da matéria, sendo indestrutível e imutável, e a combinação dos átomos formaria todos os corpos. Entre os átomos que compunham as coisas havia o vazio absoluto, o vácuo, e, portanto, tudo na Natureza poderia ser entendido a partir de dois princípios fundamentais: átomo e vazio.

O conceito da impossibilidade do vácuo seria retomado, não muito tempo depois, por outro filósofo grego, Aristóteles (ca. 384 - 322 a. C). Para Aristóteles tudo que existia era proveniente dos cinco elementos: água, fogo, terra, ar e éter ${ }^{[3]}$, sendo os quatro primeiros denominados elementos principais e o quinto, também chamado de "quintessência", era a substância que permearia todo o Universo, de forma que algo que não fosse composto por estes elementos era um absurdo. Além disso, segundo ele, os corpos possuem tendências intrínsecas, como, por exemplo, os corpos materiais possuíam a tendência de caírem, mas sofrem resistência do meio onde se encontram e caem com menor velocidade dependendo do meio, se existisse o vácuo estes corpos não seriam resistidos e aumentariam sua velocidade infinitamente ${ }^{[4]}$, o que não seria concebível. Ao que Aristóteles teria dito: "A natureza tem horror ao vácuo".

Um discípulo de Galileu Galilei (1564 - 1642) de nome Evangelista Torricelli (1608 - 1647), por volta de 1644 , realizou um simples experimento no qual foi possível obter o vácuo. Utilizando um tubo de vidro contendo mercúrio até a borda e fechado em uma das extremidades ele tapou a extremidade aberta e colocou o tubo em um recipiente contendo mercúrio. Ao soltar a extremidade aberta com o tubo imerso no recipiente notou que a coluna de mercúrio sofria uma queda e descia até que estivesse a aproximadamente $76 \mathrm{~cm}$ acima do nível de mercúrio do recipiente (conforme ilustrado na Figura 2).

Torricelli verificou que o motivo do mercúrio descer somente até que estivesse a aproximadamente $76 \mathrm{~cm}$ é que nesta altura o peso da coluna de mercúrio era equilibrado pela força exercida pela pressão atmosférica. Mas o resultado mais impressionante obtido era que o espaço gerado na parte de cima da coluna continha tão pouco ar ou matéria que poderia ser considerado como sendo o vácuo. Além disso, o "vácuo" formado poderia ser mantido pelo tempo que se desejar, de forma que Torricelli demonstrou que a natureza não tem tanto "horror" ao vácuo como afirmava a teoria aristotélica.

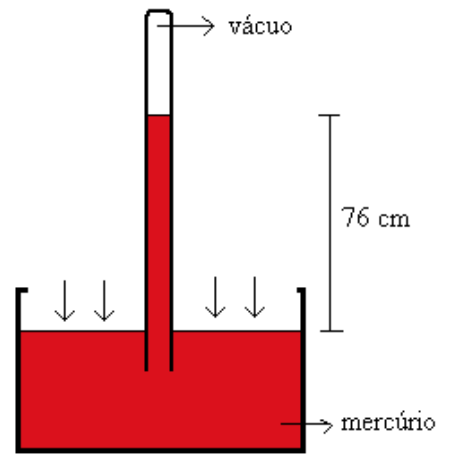

Figura 2. Experiência de Torricelli do tubo de mercúrio.

Outra experiência a respeito da obtenção do vácuo foi realizada por um cientista amador de nome Otto Von Guericke (1602 - 1686), em 1654; Ele utilizou uma bomba de vácuo, que nada mais é que um dispositivo utilizado para retirar ar de um recipiente, para remover o ar de dentro de dois hemisféricos metálicos ocos, unidos de forma meticulosa. Posteriormente ele amarrou uma corda a cada um dos hemisféricos conectadas na outra extremidade a oito cavalos cada um (ilustrado na Figura 3), de forma que estes puxaram a corda, cada grupo em uma direção, e não conseguiram separar os hemisféricos. $\mathrm{O}$ fato de não ter ar no interior dos hemisféricos unidos fez com que a pressão atmosférica mantivesse-os unidos de forma que mesmo dezesseis cavalos não conseguiram separá-los, mostrando assim que no interior dos hemisféricos havia se formado o "vácuo". Posteriormente foram abertas as válvulas dos hemisféricos para que o ar entrasse separando-os instantaneamente ${ }^{[5]}$.

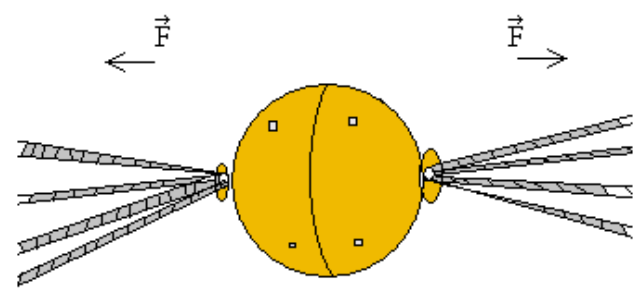

Figura 3. Experiência de Guericke dos hemisféricos.

No século XIX, o desenvolvimento do eletromagnetismo e as novas concepções acerca das características ondulatórias da luz, devido às pesquisas de James Clerk Maxwell (1831 - 1879), fez com que a ideia do vácuo contendo "algo" fosse retomada. A descoberta de que a luz era uma onda eletromagnética exigia, segundo os cientistas da época, que existisse um meio no qual ela pudesse se propagar, de 
forma que foi proposta a ideia de um meio conhecido como éter, que seria uma espécie de substância que permearia todo o espaço. Entretanto, a teoria do éter veio por água abaixo com as experiências de Albert Abraham Michelson (1852 - 1931) e Edward Morley (1838 - 1923), em 1887, que realizaram um experimento para medir a velocidade da luz em relação ao éter e ao mesmo tempo provar a sua existência. Mas, resultados negativos indicaram que tal meio muito provavelmente não existiria dada a precisão que o experimento fornecia e a falha na tentativa de sua medição ${ }^{[6]}$.

O próximo passo importante na compreensão do que é de fato o vácuo foi dado pelo físico alemão Max Planck (1858 - 1947). Em 1911, Planck [7] apresentou seu trabalho sobre a "nova hipótese da radiação" em um encontro da Sociedade de Física Alemã. Neste trabalho, ele propõe que a emissão de energia radioativa se dá de modo quantizado, isto é, de forma discreta, enquanto que a absorção de energia radioativa é de modo contínuo, diferente de sua hipótese anterior no qual emissão e absorção eram quantizados. Como consequência dessa suposição, resultados posteriores ${ }^{[8]}$ mostraram que a energia de um Oscilador Harmônico Quântico (Equação 1), a temperatura de zero Kelvin, o estado de mínima energia ou estado fundamental, diferente da situação clássica não é zero (tal energia é obtida fazendo-se $n=0$ na Equação 1). Tal energia foi nomeada de energia residual por Planck, sendo denominada posteriormente de energia de ponto de zero.

$$
E_{n}=\left(n+\frac{1}{2}\right) \hbar \omega
$$

Com o desenvolvimento da nova mecânica dos quanta, Werner Heisenberg (1901 - 1976), Pascual Jordan (1902 - 1980) e Max Born (1882 - 1970), os fundadores da mecânica matricial, descobriram e publicaram em um artigo de 1925 e 1926 que o vácuo quântico pode ser entendido como um conjunto de osciladores harmônicos, campos eletromagnéticos oscilantes, cada um possuindo uma frequência $\omega_{k} \mathrm{e}$ com energia dada por ${ }^{[9]}$ :

$$
E_{v}=\sum_{k} \frac{1}{2} \hbar \omega_{k}
$$

Tomando todos os pontos do espaço livre obtém-se um resultado impressionante: somando todos os osciladores possíveis chega-se à conclusão que a energia do vácuo é infinita, já que o número de frequência que uma cavidade pode possuir é infinito. Este resultado é alvo de entusiasmo para uns e questionamentos infindáveis para outros, afinal se a energia do vácuo é infinita talvez exista alguma forma de usufruir dela ${ }^{[10]}$, entretanto por outro lado a consequência da existência da própria energia de ponto zero associada ao vácuo quântico aparentemente torna-se um problema para a cosmologia no chamado "problema da constante cosmológica"; A densidade de energia do vácuo pode ser obtida diretamente a partir da soma de três termos: da constante cosmológica "nua", situação no qual o espaço estaria na ausência de quaisquer partículas e a única força presente seria a de gravidade; das flutuações quânticas, devido aos campos do Modelo Padrão associado aos pares de partículas virtuais que surgem espontaneamente e interagem brevemente desaparecendo em seguida; e um último termo incluído com a função de representar as contribuições para a densidade de energia do vácuo devido a partículas ou interações desconhecidas. O problema surge devido ao fato que experimentalmente verifica-se que a densidade de energia total do vácuo é menor que um valor da ordem de $10^{-47} G e V^{4}$, enquanto que as predições experimentais indicam que o seu valor é muito maior que este, da ordem de $10^{71} \mathrm{Ge} V^{4}$. Para este valor de densidade do vácuo o Universo que conhecemos seria drasticamente diferente, de forma que há a necessidade de que os termos referentes à constante cosmológica e as flutuações quânticas sejam cancelados por um termo desconhecido da ordem de $10^{118} \mathrm{GeV} \mathrm{V}^{4}$. O que mais intriga é qual mecanismo físico da Natureza seria responsável por tal cancelamento ${ }^{[11]}$.

\section{O Efeito Casimir Estático}

Nos Laboratórios Philips, Casimir trabalhava, juntamente com Dirk Polder (1919 - 2001), no estudo de soluções coloidais, que nada mais são que sistemas nos quais as partículas são pequenas demais (da ordem de um nanômetro) para serem visualizadas a olho nu, e buscava compreender quais eram as forças que provocavam a estabilidade destas soluções. Mais precisamente, estudava a ação de forças moleculares em relação a grandes distâncias. Em uma visita à Dinamarca, que ocorreu em 1947, Casimir pôde conversar com Niels Bohr (1885-1962) e mencionou ao mesmo os resultados de sua pesquisa, Bohr teria resmungado algo em relação à energia de ponto zero e isto foi mais do que o suficiente para colocá-lo em uma nova direção. Abaixo segue um trecho de uma carta privada escrita por Casimir em março de 1992 no qual ele comenta como foi a conversa que teve com Bohr ${ }^{[9]}$ :

"Eu mencionei os meus resultados para Niels Bohr, durante uma caminhada. Isso é bom, disse ele, isto é algo novo. Eu disse a ele que eu estava intrigado com a forma extremamente simples das expressões para a interação a distâncias muito grandes e ele murmurou algo sobre a energia de ponto zero. Isso foi tudo, mas colocou-me em uma nova direção."

Casimir pesquisou e calculou que havia uma pequena, mas considerável, força de atração entre duas placas paralelas, condutoras e eletricamente 
neutras e verificou que essa força correspondia às variações da energia de ponto zero, isto é, a energia proveniente do vácuo quântico: Este fenômeno é o Efeito Casimir. Vale ressaltar que o mais importante da descoberta realizada por Casimir não é em si o fato de existir uma força entre duas placas eletricamente neutras, mas sim o fato dessa força ser devida às flutuações da energia de ponto zero, o que indica que o vácuo desempenha um papel importante nas interações moleculares de forma que ele não pode mais ser ignorado. A força que Casimir obteve para duas placas condutoras paralelas e eletricamente neutras de áreas $A$ separadas por uma distância $d$ foi ${ }^{[1]}$ :

$$
F=-\frac{A \pi^{2} \hbar c}{240 d^{4}}
$$

o $\hbar$ é a constante reduzida de Planck e $c$ é a velocidade da luz no vácuo. Como nota-se pela equação (3), a força é relativamente pequena se comparada, por exemplo, com a força gravitacional e elétrica, regidas respectivamente pela Lei da Gravitação Universal e Lei de Coulomb, que decaem com o quadrado da distância; Afinal, a força de Casimir diminui com a quarta potência ao aumentar a distância de separação das placas. Na seção seguinte apresentamos de modo simplificado um método de cálculo da força de Casimir.

\section{Cálculo da força de Casimir}

A demonstração do cálculo da força de Casimir será apresentada tal como realizado por Milonni ${ }^{[12]}$. Supomos, em uma região onde se faz o vácuo, uma caixa, vazia em seu interior, na forma de um paralelepípedo com paredes perfeitamente condutoras de lados $L_{x}=L_{y}=L$ e $L_{z}=d$. As frequências permitidas para qualquer radiação "presa" no interior da caixa são restritas a um conjunto de valores discretos, sendo que em nenhuma caixa são possíveis todas as frequências; de modo que nesta situação a energia de ponto-zero é afetada pela presença da caixa e é diferente da situação no qual a caixa não está presente. A questão que pode ser levantada é: será que esta mudança na energia de ponto-zero resulta em alguma força no interior da caixa? A resposta é sim, existe uma força (a de Casimir) na caixa.

Como sabemos as frequências possíveis no interior da caixa são dadas por:

$$
\omega_{l m n}=c\left(k_{x}^{2}+k_{y}^{2}+k_{z}^{2}\right)^{1 / 2}
$$

Ou ainda,

$$
\omega_{l m n}=\pi c\left(\frac{l^{2}}{L^{2}}+\frac{m^{2}}{L^{2}}+\frac{n^{2}}{d^{2}}\right)^{1 / 2}
$$

onde $l, m$ e $n$ são valores inteiros positivos e zero. A energia de ponto-zero na caixa é, portanto:

$$
E_{0}(d)=\sum_{l m n}^{\prime}(2) \frac{1}{2} \hbar \omega_{l m n}=\sum_{l m n} \hbar \pi c\left(\frac{l^{2}}{L^{2}}+\frac{m^{2}}{L^{2}}+\frac{n^{2}}{d^{2}}\right)^{1 / 2}
$$

O fator 2 é devido às duas polarizações independentes dos modos quando $l, m, n \neq 0$, e o símbolo de apóstrofo depois do somatório refere-se ao fato de que um fator de meio deve inserido se um destes valores inteiros for zero, pois dessa forma teremos apenas uma polarização independente.

Nós estamos interessados no caso em que $L>d$, onde podemos substituir o somatório sobre $l$ e $m$ por uma integral:

$$
E_{0}(d)=\frac{\hbar c L^{2}}{\pi} \sum_{n} \int_{0}^{\infty} d x \int_{0}^{\infty} d y\left(x^{2}+y^{2}+\frac{\pi^{2} n^{2}}{d^{2}}\right)^{1 / 2}
$$

Se $d$ é arbitrariamente grande, o somatório sobre $n$ pode ser substituído por uma integral e a energia de ponto-zero pode ser escrita como:

$$
E_{0}(\infty)=\frac{\hbar c L^{2}}{\pi} \int_{0}^{\infty} d x \int_{0}^{\infty} d y \int_{0}^{\infty} d z\left(x^{2}+y^{2}+z^{2}\right)^{1 / 2}
$$

A Energia Potencial do sistema das placas quando separadas por uma distância $d$ é $U(d)=E_{0}(d)-E_{0}(\infty)$. Assim, a energia necessária para trazer as placas de uma grande separação para uma separação de $d$ é dada por:

$$
\begin{aligned}
U(d)=\frac{\hbar c L^{2}}{\pi^{2}} & {\left[\sum_{n} \int_{0}^{\infty} d x \int_{0}^{\infty} d y\left(x^{2}+y^{2}+\frac{\pi^{2} n^{2}}{d^{2}}\right)^{1 / 2}\right.} \\
- & \left.\frac{d}{\pi} \int_{0}^{\infty} d x \int_{0}^{\infty} d y \int_{0}^{\infty} d z\left(x^{2}+y^{2}+z^{2}\right)^{1 / 2}\right]
\end{aligned}
$$

Colocando em coordenadas polares e fazendo uma simples transformação de variável de integração, temos que:

$$
\begin{array}{r}
U(d)=\frac{\hbar c L^{2}}{\pi^{2}} \frac{\pi}{2}\left[\sum_{n=0}^{\infty} \int_{0}^{\infty} r d r\left(r^{2}+\frac{\pi^{2} n^{2}}{d^{2}}\right)^{1 / 2}\right. \\
\left.-\frac{d}{\pi} \int_{0}^{\infty} d z \int_{0}^{\infty} r d r\left(r^{2}+z^{2}\right)^{1 / 2}\right] \\
\begin{aligned}
U(d)=\frac{\hbar c L^{2}}{4 \pi} \frac{\pi^{3}}{d^{3}}\left[\sum_{n=0}^{\infty} \int_{0}^{\infty} d x\left(x+n^{2}\right)^{1 / 2}\right. \\
\left.-\int_{0}^{\infty} d z \int_{0}^{\infty} d x\left(x+z^{2}\right)^{1 / 2}\right]
\end{aligned}
\end{array}
$$

Esta é a diferença entre duas energias infinitas (ponto-zero). Podemos reescrever essa energia como: 


$$
U(d)=\frac{\pi^{2} \hbar c L^{2}}{4 d^{3}}\left[\frac{1}{2} F(0)+\sum_{n=1}^{\infty} F(n)-\int_{0}^{\infty} d z F(z)\right]
$$

onde, $\operatorname{com} u=n$ ou $z$ :

$$
F(u) \equiv \int_{0}^{\infty} d x\left(x+u^{2}\right)^{1 / 2}
$$

Há várias maneiras de avaliar a diferença entre dois infinitos. Nós devemos empregar a fórmula de Euler-Mclaurin

$$
\sum_{n=1}^{\infty} F(n)-\int_{0}^{\infty} d z F(z)=-\frac{1}{2} F(0)-\frac{1}{22} F^{\prime}(0)+\frac{1}{720} F^{\prime \prime \prime}(0) \ldots
$$

para a diferença entre o somatório e o integral, onde os apóstrofos representam derivadas. Note que $F^{\prime}(z)=-2 z^{2}$, vemos também que $F^{\prime}(0)=0$ e $F^{\prime \prime \prime}(0)=-4$, e as derivadas de ordem maiores desaparecem. Assim,

$$
\sum_{n=1}^{\infty} F(1)-\int_{0}^{\infty} d z F(z)=-\frac{1}{2} F(0)-\frac{4}{720}
$$

E,

$$
U(d)=\frac{\pi^{2} \hbar c L^{2}}{4 d^{3}}\left(-\frac{4}{720}\right)=-\frac{\pi^{2} \hbar c L^{2}}{720 d^{3}}
$$

Isto implica que a força (de Casimir) é:

$$
F(d)=U^{\prime}(d)=-\frac{A \pi^{2} \hbar c}{240 d^{4}}
$$

onde $A=L^{2}$ é a área lateral da caixa.

\section{Verificação experimental do Efeito Casimir}

A primeira verificação experimental do Efeito Casimir veio no ano de 1958, dez anos após o artigo publicado por Casimir, através do holandês Marcus Sparnaay nos Laboratórios Philips em Eindhoven ${ }^{[13]}$. Devido à delicadeza com que o experimento deveria ser realizado e também devido à magnitude da força, que como visto anteriormente (Equação 2) diminui com a quarta potência com o aumento da distância de separação entre as placas, Sparnaay, embora tenha conseguido medir a força, não conseguiu obtê-la com precisão, e seus resultados demonstraram grande margem de erro experimental. Mesmo uma mínima quantidade de carga no interior da câmara contendo o vácuo ou mesmo uma pequena divergência no paralelismo entre as placas seria o suficiente para obter uma força no interior da câmara que não fosse a do
Efeito Casimir, daí a dificuldade em realizar tal medição.

O próximo passo na detecção do Efeito Casimir foi dado no Laboratório Nacional de Los Alamos, em 1997 , por Steve. K. Lamoreaux que verificou a existência do efeito utilizando uma placa metálica plana e outra esférica variando a distância entre elas de 0.6 micrômetros a 6.0 micrômetros, de forma que foi possível eliminar consideravelmente a incerteza devido à dificuldade de alinhar as placas planas. Lamoreaux ${ }^{[14]}$ obteve em suas medidas um erro experimental de $5 \%$, de forma que tal precisão foi mais do que suficiente para legitimar a existência do Efeito Casimir.

Medidas mais precisas foram obtidas posteriormente, como a de Anushree Roy e Umar Mohideen [15], em 1998, na Universidade da Califórnia. Eles obtiveram um resultado com uma incerteza de apenas $1 \%$.

Recentemente, em 2009, J. N. Munday, F. Capasso e V. A. Parsegian ${ }^{[16]}$ verificaram experimentalmente a existência do Efeito Casimir de caráter repulsivo, previsto teoricamente pelo físico russo Evgeny Lifshitz (1915 - 1985); Este fenômeno é às vezes chamado de levitação quântica.

\section{Conclusão}

De tempos em tempos, a Física é tida como estando completa e é comum encontrar afirmações que nada de novo será descoberto. Entretanto, contrariando tal visão, a natureza sempre nos mostra novos fenômenos como o Efeito Casimir, discutido neste trabalho.

Outro fenômeno de fundamental importância é o Efeito Casimir dinâmico, no qual é estudada a força de Casimir existente na situação em que ocorre o movimento no arranjo geométrico das cavidades que aprisionam uma região do vácuo. Uma vez que o vácuo é entendido agora como sendo um agente real, no sentido de ser influente em sistemas físicos, podemos verificar como é a sua reação quando submetido a oscilações das paredes que o rodeiam ${ }^{[10]}$. E esta é somente mais uma das etapas na evolução das ideias propostas inicialmente por Hendrik Casimir, muitas outras estão por vir e o desenvolvimento de teorias correlacionadas continua a todo vapor.

A previsão realizada por Casimir da força de atração entre as placas paralelas, condutoras e eletricamente neutras, e posterior estudo das flutuações da energia de ponto zero, surgiu como componente fundamental para a compreensão de que o vácuo não é o vazio absoluto e que este possui enorme importância no estudo das interações moleculares. Como ressaltado por Milonni ${ }^{[9]}$ :

"A teoria quântica de campos nos ensinou que o vácuo não é um tranquilo estado do nada, mas um estado quântico com flutuações e consequências físicas. Talvez o uni- 
verso, ele mesmo, tenha surgido de uma flutuação quântica."

\section{Referências}

[1] Casimir, H. B. G. On the attraction between two perfectly conducting plates (Sobre a atração entre duas placas perfeitamente condutoras). Proceedings of the Royal Netherlands Academy of Arts and Sciences. Vol. 51, p. 793-795. (1948).

[2] Parmênides. Da Natureza. Tradução de Fernando Santoro, Laboratório OUSIA, Rio de Janeiro, RJ. (2007).

[3] Peduzzi, L. O. Q. Física Aristotélica: Porque não considerá-la no ensino da Mecâni$c a$ ? Caderno Catarinense de Ensino de Física. Vol. 13, p. 48-63. (1996).

[4] Stempniak, R. A. A Ciência e a Tecnologia do Vácuo. Faculdade de Ciências Aplicadas de São José dos Campos, SP. (2002).

[5] Cougo-Pinto, M. V; Farina, C. and Tort, A. C. O Efeito Casimir. Revista Brasileira de Física, Vol. 22, p. 122-132. (2000).

[6] Caruzo, F; Oguri, V. Física Moderna. Editora Campus, Rio de Janeiro. (2006).

[7] Planck, M. Über die Begründung des Gesetzes der Schwarzen Strahlung (Sobre a justificação da lei da radiação de corpo negro) Annalen der Physik. Vol. 342, p. 642656. (1912).

[8] Heisenberg, W. Über Quantentheriorische Undeutung Kenmatischer und Mechanischer Beziehungen (Sobre a interpretação quântica das relações cinemáticas e mecânicas). Zeitschrift für Physik. Vol. 33, p. 879-893. (1912).

[9] Milloni, P. W. The Quantum Vacuum: an introduction to quantum electrodynamics (O Vácuo Quântico: uma introdução à eletrodinâmica quântica). Academic Press, Los Alamos, New Mexico. (1993).

[10] Andreata, M. A. Processos quânticos em cavidades com paredes vibrantes. $71 \mathrm{f}$. Dissertação (Mestrado em Física) - Centro de Ciências Exatas e de Tecnologia, Universidade Federal de São Carlos, São Carlos. (2000).
[11] Weinberg, S. The cosmological constant problem (O problema da constante cosmológica), Reviews of Modern Physics. Vol. 61, p. 1-23. (1989).

[12] Milloni, P. W. Casimir Forces (Forças de Casimir). Contemporary Physics, Vol. 33. p. 313-322. (1992).

[13] Sparnaay, M. J. Measurements of Attractive Forces between Flat Plates (Medidas das forças atrativas entre placas planas). Physica, Vol. 24. p. 6-10. (1958).

[14] Lamoreaux, S. K. Demonstration of the Casimir Forces in the 0.6 to $6 \mu \mathrm{m}$ (Demonstração da força de Casimir no intervalo de 0,6 a $6 \mu \mathrm{m})$. Physical Review Letters. Vol. 78, p. 5-8. (1997).

[15] Mohideen, U. and Roy, A. Precision Measurent of the Casimir forces from 0.1 to $0.9 \mu \mathrm{m}$ (Medida precisa da força de Casimir no intervalo de 0,1 a $0,9 \mu \mathrm{m}$ ). Physical Review Letters. Vol. 81, p. 4549-4552. (1998).

[16] Munday, J. N; Capasso, F. and Parsegian, V. A. Measured long-range repulsive Casimir-Lifshitz forces (Medidas das forças repulsivas de longo alcance de CasimirLifshitz). Nature, Vol. 457, p. 170-173. (2009). 